Check for updates

Cite this: RSC Adv., 2017, 7, 56489

Received 7th November 2017

Accepted 4th December 2017

DOI: $10.1039 / c 7 r a 12201 b$

rsc.li/rsc-advances

\section{Light- and heat-triggered reversible luminescent materials based on polysiloxanes with anthracene groups $\uparrow$}

\author{
Dongdong Han, Hang Lu, Wensi Li, Yonghao Li and Shengyu Feng (iD *
}

\begin{abstract}
In this study, reversible silicone elastomers were successfully developed by light-triggered dimerization and heat depolymerization of the anthryl groups. Polysiloxanes with anthryl groups were prepared from poly(aminopropylmethylsiloxane) (PAPMS) with electron-donating (9-anthracenylmethyl acrylate) and electron-withdrawing (anthracene-9-carboxylic acid) units. The cross-linking networks were formed with the via $4 \pi-4 \pi$ photo-cycloadditions of the anthryl groups upon the UV light excitation (365 nm). 9Anthracenylmethyl acrylate or anthracene-9-carboxylic acid efficiently dimerized through the photodimerization of the anthryl groups in the organic solvents, which was proven by UV-vis spectra, NMR spectra, and LC/MS. The covalent bonds between pendant anthryl groups were cleaved after heating at $120{ }^{\circ} \mathrm{C}$. Furthermore, repeatable dimerization-depolymerization conversion was confirmed. In addition, for the first time, we found that the sunlight can also initiate the cycloaddition, which was "greener" and more environment-friendly. The green luminescence was observed from the PAPMS-1 film instead of the quenching effect caused by aggregation after the cycloaddition. Thus, a colorful UV-light emitting diode (LED) cell was obtained by coating the films on the commercially available LED cell.
\end{abstract}

\section{Introduction}

Silicone elastomers are widely used as safe and durable materials for domestic and industrial applications because of their resistance to weathering, high and low temperatures, radiation, and fire. ${ }^{1-5}$ However, traditional silicones are normally thermoset materials, which are prepared by three main methods: (1) Pt-catalyzed hydrosilylation between $\mathrm{Si}-\mathrm{H}$ and vinyl groups; (2) condensation reaction between Si-OR and -OH; (3) free-radical cross-linking. ${ }^{6}$ Recently, many classical reactions such as thiolene "click" reactions, ${ }^{7}$ Michael addition, ${ }^{8}$ and $[2+2]$ photocycloaddition ${ }^{9}$ were utilized to develop self-healing, thermosensitive, fluorescent, or reversible silicone elastomers. For example, Brook et al. prepared a thermo-sensitive silicone elastomer via $[2+2]$ photo-cycloaddition of coumarin. Coumarin is UV-sensitive and can undergo a reversible reaction upon UV irradiation. ${ }^{9}$ In addition, Brook et al. prepared a selfassociating silicone elastomer based on azide/alkyne cycloaddition reactions. ${ }^{\mathbf{1 0}}$ In our group, highly sensitive stimuliresponsive polysiloxanes (SPSis) were prepared via a facile, efficient, and catalyst-free aza-Michael addition of

Key Laboratory of Special Functional Aggregated Materials, Key Laboratory of Colloid and Interface Chemistry (Shandong University), Ministry of Education, School of Chemistry and Chemical Engineering, Shandong University, Jinan 250100, P. R. China. E-mail: fsy@sdu.edu.cn

$\dagger$ Electronic supplementary information (ESI) available. See DOI: 10.1039/c7ra12201b poly(aminopropylmethylsiloxane) (PAPMS) with $\mathrm{N}$-isopropylacrylamide. ${ }^{11}$ However, these methods have some drawbacks, including potential explosiveness of the azide monomers, the unpleasant smell of thiol-enes, the high costs of the catalysts, and the inherent difficulties of the $[2+2]$ photocross-linking reactions. Thus, it is desirable to develop a simple, efficient, and catalyst-free system to prepare functional and cross-linked silicones.

In spite of many synthetic methods used to produce silicone materials, there are few reports about photo-cross-linking reactions without a photo-initiator or catalysts. Anthracene is commonly used in photo-responsive compounds, which can form covalent bonds through photo-cycloaddition. ${ }^{12-22}$ Anthracene groups undergo $[4+4]$ photo-dimerization when irradiated by UV light $(\lambda>300 \mathrm{~nm})$ and can be reversed to the original monomers via exposure to a higher energy UV light $(\lambda<300 \mathrm{~nm})$ (Scheme 1$)^{23}$ or heating to $100-130{ }^{\circ} \mathrm{C}^{24,25}$ Xie et al. successfully obtained shape-memory materials by dangling photoresponsive anthracene groups from different polymer chains. ${ }^{23,26,27}$

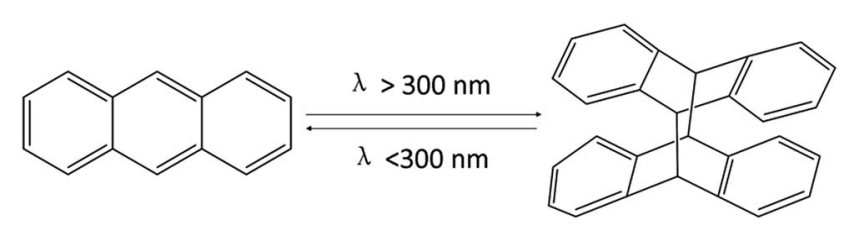

Scheme 1 Reversible photo-dimerization reaction of anthracene groups. ${ }^{23}$ 
However, there are few reports on reversible silicone elastomers based on the anthracene groups.

Based on the above discussion, we supposed that the stimuli or environmentally responsive silicone elastomers could be obtained from the reaction of polysiloxanes and the anthracene groups.

In this study, we appended the anthryl groups to polysiloxanes with different anthracene derivatives and conducted a comprehensive examination on the reversible conversion process with heat. It is well-known that the dimerization or dissociation rate of anthryl dimers depends on the substituents on the anthracene ring. ${ }^{28}$ Thus, we synthesized silicone elastomers by combining poly(aminopropylmethylsiloxane) (PAPMS) with two derivatives of 9-substituted anthracene, in which one was linked with an electron-donating group (9-anthracenylmethyl acrylate, PAPMS-1) and the other was linked with an electron-withdrawing substituent (anthracene-9-carboxylic acid, PAPMS-2). The prepolymer of PAPMS-1 was successfully prepared from 9-anthracenylmethyl acrylate and PAPMS through an aza-Michael reaction, which is operated at mild reaction conditions, has a high efficiency, does not require a catalyst, and produces no by-products. However, the prepolymer of PAPMS-2 was prepared via "salt-forming vulcanization"6 by neutralizing PAPMS with anthracene-9-carboxylic acids to yield an ion-association complex. Then, light- and heattriggered reversible silicone elastomers were prepared via $[4+$ 4] photo-dimerization of the pendant anthryl groups of the prepolymers. The cross-linked network was formed among the polymer chains after the polymer solution was treated with UV light (365 $\mathrm{nm})$.

Sunlight-induced photo-dimerization was applied, for the first time, in the anthracene system, thus making it a "greener" and more environment-friendly process. As expected, dimerization efficiency of anthryl groups was remarkable when the sunlight was applied in the PAPMS-1 system. The covalent bonds formed via dimerization of the pendant anthryl groups could be reversed when the polymer was heated at $120^{\circ} \mathrm{C}$. We found that the PAPMS-1 film could emit green light under UV irradiation, which made the composites suitable for optoelectronic devices applications, such as light emitting diodes (LEDs). When the polymer solution was coated on the commercially available UV-LED cells and cured in situ, the colorful LED cells could be fabricated in an economical and effective way.

\section{Experimental}

\section{Materials}

Octamethylcyclotetrasiloxane $\left(\mathrm{D}_{4}\right)$ and 3-aminopropyl-methyldiethoxysilane were obtained as the commercial products and used directly. Dichloromethane, acetic acid, petroleum, ethanol, toluene, trichloromethane, and methanol were obtained from Tianjin Fuyu Chemical Co., Ltd. 9-Anthraldehyde, acryloyl chloride and anthracene-9-carboxylic acid were supplied by Energy Chemical Technology Co., Ltd. Sodium borohydride was provided by the Tianjin Kermel Chemical Reagent Co., Ltd.

\section{Synthesis}

Synthesis of 9-anthracenemethanol. Sodium borohydride $(5.0 \mathrm{~g})$ was dissolved in ethanol $(50 \mathrm{~mL})$ and added dropwise to the reaction mixture of 9-anthraldehyde $(10.0 \mathrm{~g})$ in ethanol (50 $\mathrm{mL}$ ). The resulting mixture was sealed and then stirred for $2 \mathrm{~h}$ in an ice bath. Then, water $(25 \mathrm{~mL})$ was poured into the mixture. A yellow solid $(8.1 \mathrm{~g})$ was obtained after filtration and vacuum drying (yield $80 \%$ ).

${ }^{1} \mathrm{H}$ NMR $\left(400 \mathrm{MHz}, \mathrm{CDCl}_{3}\right): \delta 8.48(\mathrm{~s}, 1 \mathrm{H}), 8.43(\mathrm{~d}, J=9.0 \mathrm{~Hz}$, $2 \mathrm{H}), 8.04(\mathrm{~d}, J=8.4 \mathrm{~Hz}, 2 \mathrm{H}), 7.64-7.53(\mathrm{~m}, 2 \mathrm{H}), 7.49$ (dd, $J=7.9$, $7.0 \mathrm{~Hz}, 2 \mathrm{H}), 5.69$ (s, 2H).

Synthesis of 9-anthracenylmethyl acrylate. 9-anthracenemethanol $(4.50 \mathrm{~g})$ and trimethylamine $(3.0 \mathrm{~g})$ were dissolved in $25 \mathrm{~mL}$ dichloromethane (DCM). Then, acryloyl chloride (2.5 g) was added dropwise to the reaction mixture. The resulting mixture was sealed and stirred for $2 \mathrm{~h}$ in an ice bath. The solvent and the unreacted acryloyl chloride were removed under vacuum. Next, triethylamine salt was removed by column chromatography using petroleum ether-dichloromethane $(1: 1)$ as an eluent. A yellow solid (4.6 g) was obtained after vacuum drying (yield $81 \%$ ).

${ }^{1} \mathrm{H}-\mathrm{NMR}\left(400 \mathrm{MHz}, \mathrm{CDCl}_{3}\right): \delta 8.55(\mathrm{~s}, 1 \mathrm{H}), 8.39(\mathrm{~d}, J=9.0 \mathrm{~Hz}$, $2 \mathrm{H}), 8.07$ (d, $J=8.4 \mathrm{~Hz}, 2 \mathrm{H}), 7.66-7.57(\mathrm{~m}, 2 \mathrm{H}), 7.57-7.46(\mathrm{~m}$, 2H), 6.45 (dd, $J=17.3,1.4 \mathrm{~Hz}, 1 \mathrm{H}), 6.27$ (s, 2H), 6.16 (dd, $J=$ $17.3,10.4 \mathrm{~Hz}, 1 \mathrm{H}), 5.83$ (dd, $J=10.4,1.4 \mathrm{~Hz}, 1 \mathrm{H}$ ).

Synthesis of PAPMS. PAPMS was synthesized by hydrolysis and ring opening polymerization. Typically, $100 \mathrm{~g} \mathrm{D}_{4}$ and $10 \mathrm{~g}$ 3aminopropyl-methyl-diethoxysilane were added to a $250 \mathrm{~mL}$ three-neck flask equipped with a mechanical stirrer. Then, $0.053 \mathrm{~g}(0.05 \mathrm{wt} \%) \mathrm{KOH}, 1.88 \mathrm{~g}$ water, and $1.1 \mathrm{~g}$ (1.0 wt\%) DMSO were added to the mixture. The mixture was refluxed and stirred for $4 \mathrm{~h}$ at $100{ }^{\circ} \mathrm{C}$ and then ethanol and water were removed under vacuum. The resultant mixture was stirred slowly at $120{ }^{\circ} \mathrm{C}$ for $15 \mathrm{~h}$ and cooled to room temperature. $\mathrm{KOH}$ was neutralized by acetic acid. The mixture was heated to $180{ }^{\circ} \mathrm{C}$ under vacuum to remove the low-boiling components and a white viscous liquid was obtained.

${ }^{1} \mathrm{H}$ NMR $\left(400 \mathrm{MHz}, \mathrm{CDCl}_{3}\right) \delta 2.67(\mathrm{dd}, J=15.1,8.1 \mathrm{~Hz}, 2 \mathrm{H})$, 1.58-1.25 (m, 4H), 0.60-0.46 (m, 2H), 0.25-0.10 (m, 73H).

\section{Preparation of the elastomers PAPMS-1 and PAPMS-2}

For preparation of PAPMS-1, as shown in Scheme 2, 9-anthracenylmethyl acrylate and PAPMS, with a certain mole ratio, were added to a flask. The mixture was dissolved in the mixed

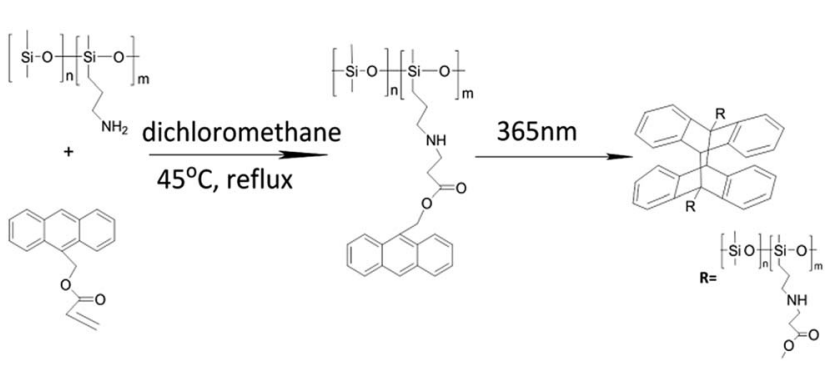

Scheme 2 Synthesis route of PAPMS-1. 
solvents of methanol $(1 \mathrm{~mL})$ and toluene $(10 \mathrm{~mL})$. Then, the resultant mixture was refluxed and stirred at $45{ }^{\circ} \mathrm{C}$. The FT-IR spectra of the mixture are presented in the ESI (Fig. S1 $\dagger$ ). As shown in Fig. $\mathrm{S} 1, \dagger$ the double bond disappeared after $8 \mathrm{~h}$, which indicated that the aza-Michael reaction was completed. The mixture (PAPMS containing anthryl groups) was stirred in an ice bath under $365 \mathrm{~nm}$ UV-irradiation for $1 \mathrm{~h}$. Then, most of the solvent was removed through distillation under reduced pressure till the resultant mixture could still be fluid. The thick precursor was placed in a Teflon mold and then the remaining solvent was slowly volatilized at room temperature.

In addition, for the first time, sunlight-induced photodimerization was attempted for anthryl silicone elastomers. A mixture of 9-anthracenylmethyl acrylate and PAPMS was irradiated by sunlight for 3 days instead of UV light. Then, most of the solvent was removed through distillation under reduced pressure till the resulting mixture could still be fluid. The thick precursor was placed into a Teflon mold, and then the remaining solvent was slowly volatilized at room temperature.

PAPMS-2 was prepared via "salt-forming vulcanization" by neutralizing PAPMS with anthracene-9-carboxylic acids to yield an ion-association complex. Typically, anthracene-9-carboxylic acids and PAPMS, in a certain mole ratio, were added to a flask. The mixture was dissolved in the mixed solvents of methanol $(1 \mathrm{~mL})$ and toluene $(10 \mathrm{~mL})$. The mixture was stirred in an ice bath upon UV-irradiation (365 nm). Fig. S2† depicts the IR spectra of anthracene-9-carboxylic acids, PAPMS, and the PAPMS-2 elastomer. The results confirmed the formation of an ion-association complex. In PAPMS-2, $-\mathrm{COOH}$ and $-\mathrm{NH}_{2}$ groups disappeared completely. Therefore, the new absorption band was observed at $1570 \mathrm{~cm}^{-1}$, which was caused by the asymmetric deformation of $-\mathrm{COO}^{-}$. This result inferred the complex formation of $-\mathrm{COO}^{-}$with $-\mathrm{NH}_{3}{ }^{+}$. The mixture was stirred in an ice bath under UV-irradiation at $365 \mathrm{~nm}$. Then, most of the mixed-solvent was removed under vacuum till the resulting mixture can still be fluid. The thick precursor was poured into a Teflon mold and the rest of the solvent evaporated naturally.

\section{Characterization and measurements}

The dimerization of the anthracene groups was accomplished by irradiating with UV light from a Spectroline Model SB-100P/ FA lamp (365 nm, $100 \mathrm{~W})$. The UV intensity was set at $4500 \mathrm{~mW}$ $\mathrm{cm}^{-2}$ at a distance of $38 \mathrm{~cm}$. The proton nuclear magnetic resonance $\left({ }^{1} \mathrm{H}-\mathrm{NMR}\right)$ spectra were recorded on a Bruker AVANCE 400 spectrometer at $25^{\circ} \mathrm{C}$ using $\mathrm{CDCl}_{3}$ (or $\mathrm{CD}_{3} \mathrm{OD}$ ) as the solvent and without tetramethylsilane as an interior label. The molecular weights were determined by an Agilent HP1100 LC-Applied Biosystems API 4000 TQ mass spectrometer (LC/ MS). The luminescence (excitation and emission) spectra of the samples were determined with a Hitachi F-7000 fluorescence spectrophotometer using a monochromated Xe lamp as an excitation source. Both excitation and emission slits were set at $5 \mathrm{~nm}$. The ultraviolet absorption (UV) spectra in dichloromethane (or toluene) solution were detected using a Beijing TU1901 double beam UV-vis spectrophotometer. Thermogravimetric analysis (TGA) was performed under $\mathrm{N}_{2}$ using a TA
SDTQ600 over a temperature range from room temperature to $800{ }^{\circ} \mathrm{C}$ with a heating rate of $10^{\circ} \mathrm{C} \mathrm{min}^{-1}$. The scanning electron microscopy (SEM) images were obtained using a Hitachi S-4800 $(7 \mathrm{kV})$. The samples were cut and coated with a thin layer of platinum before the investigation.

\section{Results and discussion}

Anthracene groups undergo dimerization ([4+4] cycloaddition reaction) when irradiated by $\mathrm{UV}(\lambda>300 \mathrm{~nm}) .{ }^{26}$ In this study, the photo-cycloaddition between the anthracene groups was accomplished through UV-irradiation at $365 \mathrm{~nm}$. In order to confirm the effectiveness of dimerization under the UV light, the control experiments were performed using 9-anthracenylmethyl acrylate or anthracene-9-carboxylic acid under the same conditions. The final products were characterized by UVvis spectrometry, ${ }^{1} \mathrm{H}-\mathrm{NMR}$, and mass spectrometry. The ${ }^{1} \mathrm{H}$ NMR spectra of the dimers are presented in Fig. 1. Compared to the spectrum of the monomer of before irradiation, few new peaks are observed in the spectrum of the resultant dimer (after the UV irradiation). For 9-anthracenylmethyl acrylate, the peaks $\mathrm{k}$ and $\mathrm{l}$ are observed at 7.0 and $6.8 \mathrm{ppm}$, respectively, after the UV-irradiation (Fig. 1a), which matched with the structure of a dimer. This result confirmed the occurrence of the dimerization reaction. For anthracene-9-carboxylic acid, the peaks $\mathrm{k}$ and $l$ are located at 7.0 and $6.9 \mathrm{ppm}$, respectively, after the UVirradiation (Fig. 1c), which showed the abundant changes in the anthracene unit signals compared with 9-anthracenylmethyl acrylate after the photo-irradiation. The results are primarily related to the substituents on the anthracene rings. However, the proton peaks of the monomer still existed after the

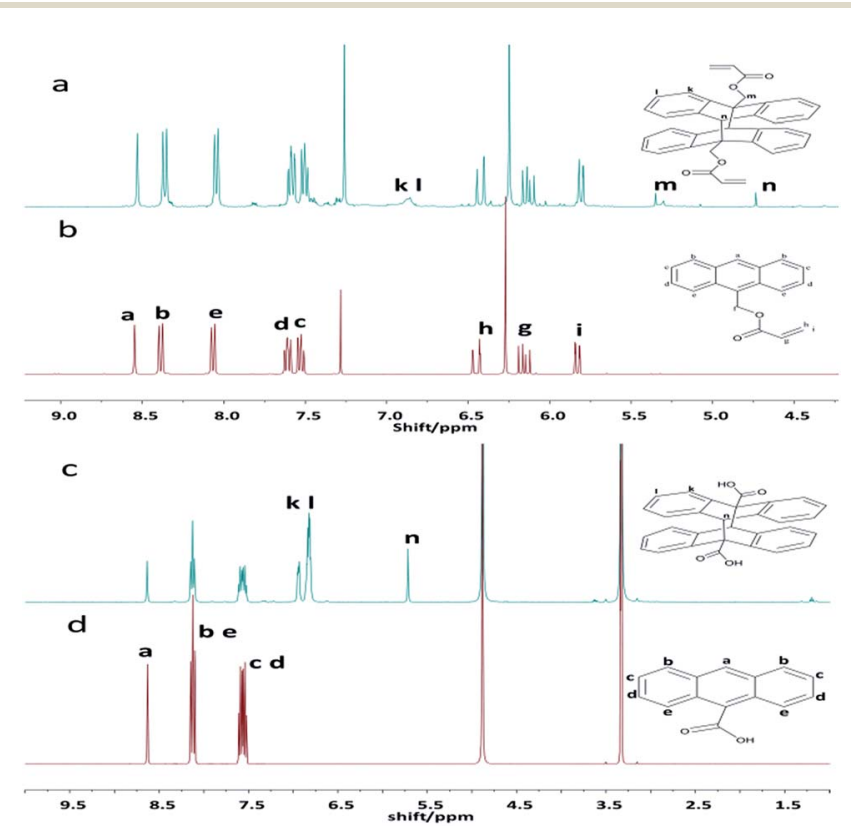

Fig. $1{ }^{1} \mathrm{H}$-NMR spectra of the anthracene derivatives after ((a) and (c)) and before ((b) and (d)) the [4 + 4] photo-cycloaddition of 9-anthracenylmethyl acrylate and anthracene-9-carboxylic acid, respectively, upon a UV irradiation at $365 \mathrm{~nm}$ for $1 \mathrm{~h}$. 
irradiation, which indicated that the dimerization reaction was incomplete. The mass spectra also showed a dimer peak and a monomer peak in the final product after the irradiation as shown in Fig. S3 and S4. $\dagger$ The results obtained from the ${ }^{1} \mathrm{H}-$ NMR and mass spectra demonstrated that only small amount of the monomer can undergo a cycloaddition reaction upon UV irradiation at $365 \mathrm{~nm}$.

The main absorption peak of anthracene in the UV-vis spectra is between 300 and $400 \mathrm{~nm}$. Upon dimerization at the 9 and 10 positions of the anthracene moieties, the conjugated $\pi$-system was broken, which led to a depletion of the characteristic UV absorption bands of the anthracene groups. As shown in Fig. 2, the absorption intensity decreased upon the UV-irradiation, which indicated that the cycloaddition had occurred. In addition, the progress of the dimerization reaction was estimated by the proportion of the decrease in the absorbance compared to its original value before the irradiation, using the equation $\left(A_{0}-A\right) / A_{0}$, where $A_{0}$ and $A$ represent the absorption intensity at $368 \mathrm{~nm}$ before and after the irradiation, respectively ${ }^{29}$ It is well-known that the rate of this dimerization depends on the substituents of the anthracene ring and the rate of reaction with the electron-withdrawing substituents is higher than that with the electron-donating substituents. As shown in Fig. 2, the efficiency of dimerization of the monomers was $25 \%$ and $35 \%$, respectively. The results achieved by UV-vis spectrometry, ${ }^{1} \mathrm{H}-\mathrm{NMR}$, and mass spectrometry proved that the dimerization reaction easily occurs when the substituent on the anthracene ring is an electron-withdrawing group.

The photo-dimerization reaction $\left(\left[\begin{array}{l}4+4\end{array}\right]\right.$ photocycloaddition) in the polymer solution can also be determined by UV-vis spectroscopy. Fig. S5 $\dagger$ shows the UV-vis spectra of the PAPMS-1 solution and the PAPMS-2 solution before and after the UV-irradiation. Although the absorption intensity of both polymer solutions decreased after the UV-irradiation, the absorption of the PAPMS-2 solution decreased more than that of the PAPMS-1 solution, which was primarily attributed to the substituents of the anthracene rings. The absorption intensity of the polymer solution was related to the exposure time as illustrated in Fig. 3. The polymer solution before the irradiation has the strongest absorption intensity in the range of 300$400 \mathrm{~nm}$. The intensity of the absorption peak decreased with the increase in the time of irradiation, which indicated that the
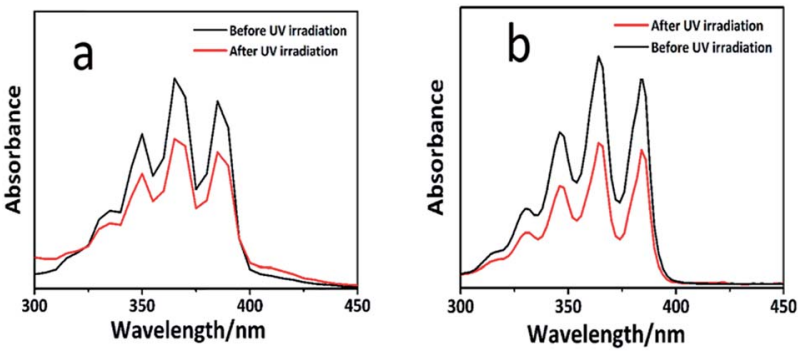

Fig. 2 (a) UV-vis spectra of 9-anthracenylmethyl acrylate before (top) and after (bottom) irradiation (365 nm); (b) UV-vis spectra of anthracene-9-carboxylic acid before (top) and after (bottom) the irradiation (365 nm).
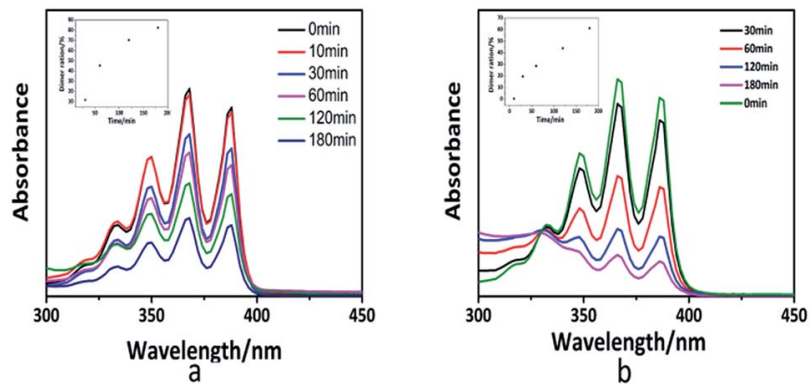

Fig. 3 UV-vis spectra and dimer ration (insets) of the PAPMS-1 solution (a) and PAPMS-2 solution (b) with different exposure times $(365 \mathrm{~nm})$

efficiency of the photo-dimerization increased with the increase in the exposure time (insets in Fig. 3). For the same irradiation time, the absorption intensity of PAPMS-2 decreased more than that of the PAPMS-1. Our data have further confirmed that the efficiency of photo-dimerization is higher if the substituent on the anthracene ring is an electron-withdrawing group.

Polysiloxanes containing pendant anthryl groups are expected to show an ascendancy of the controllable reversibility and reactivity as well as avoid the formation of by-products. The reversibility of dimerization is favorable for the degradation and the recovery of the materials. In the previous reports, the cleavage of the photo-dimers can be either optically triggered by short-wavelength UV irradiation $(254 \mathrm{~nm})$ or thermally-induced bond scission. ${ }^{30,31}$ However, compared with the thermal degradation, the UV irradiation may cause some side reactions, which limited the use of this reversible system. In order to investigate the reversible formation of the photo-cross-linking network, a heat-triggered de-cross-linking route with a thermal annealing step at $120^{\circ} \mathrm{C}$ was designed. UV-vis spectroscopy was utilized to monitor the reversible dimerization of the anthracene groups. The light- $(\lambda=365 \mathrm{~nm}, 60 \mathrm{~min})$ and heat-triggered $\left(120{ }^{\circ} \mathrm{C}\right)$ reaction, denoted as a reversible cycle, was operated. As illustrated in Fig. 4a and b, the absorption intensity of the PAPMS-1 solution and the PAPMS-2 solution decreased after the irradiation, but almost recovered to the original value after the thermal process. These results indicated that some of the anthryl groups could form dimers after the UV irradiation and the dimers were cleaved to form the anthryl groups again after the thermal treatment. Nevertheless, more time was needed for the PAPMS1 solution for de-cross-linking $\left(120^{\circ} \mathrm{C}, 240 \mathrm{~min}\right)$ compared to that for the PAPMS-2 solution $\left(120^{\circ} \mathrm{C}, 180 \mathrm{~min}\right)$. In addition, we detected the reversible property of the films as shown in Fig. S6. $\uparrow$ The results also revealed the dimerization upon UVirradiation at $365 \mathrm{~nm}$ and the bond cleavage of the anthracene dimers by heat treatment $\left(120^{\circ} \mathrm{C}\right)$. Furthermore, the model experiment was performed. As shown in Fig. 4d, the prepolymer PAPMS-2 was a viscous liquid at room temperature, whereas it was solidified when exposed to a $365 \mathrm{~nm}$ light. It is surprising that PAPMS-2 appeared to be a liquid when heated at $120^{\circ} \mathrm{C}$ and changed into the solid sample when irradiated at $365 \mathrm{~nm}$. In addition, the reversible cycle (Fig. S7 $\dagger$ ) of PAPMS-1 was longer than that of PAPMS-2. It is reported that the rate of this 

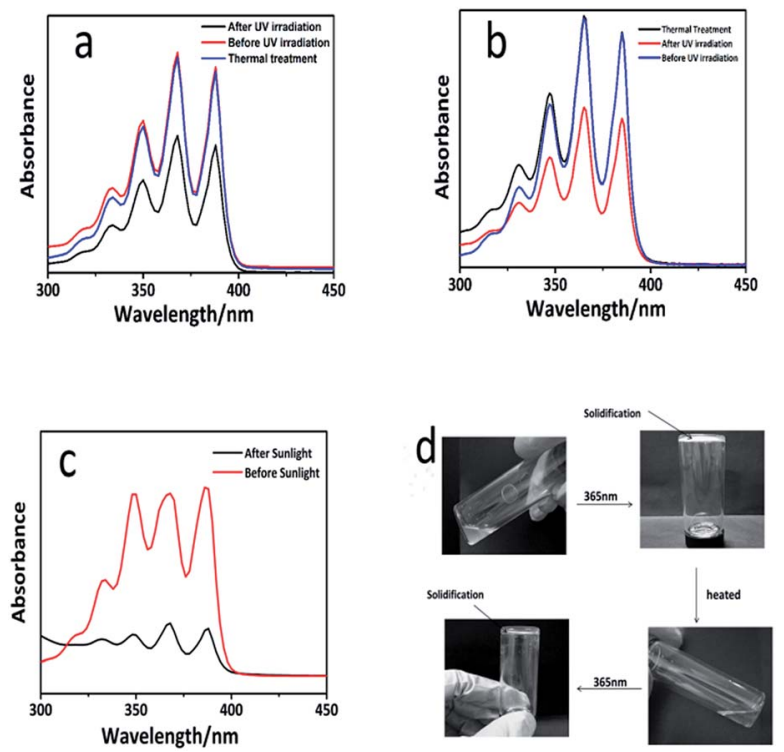

Fig. 4 (a) UV-vis spectra of the PAPMS-1 solution in different processes; (b) UV-vis spectra of PAPMS-2 solution in different processes; (c) UV-vis spectra of the anthracene modified polysiloxane solution before and after the irradiation (sunlight); (d) illustrations of PAPMS-2 at $365 \mathrm{~nm}$ upon repeating irradiation and heating cycles.

dissociation, which is caused by anthracene dimers that dissociate cleanly at the elevated temperatures through a diradical transition state, depends on the substituents on the anthracene ring. ${ }^{32}$ As expected, PAPMS- 2 could be easily depolymerized than PAPMS-1 because the anthracene in PAPMS-2 was linked to an electron-withdrawing group.

Sunlight is a green energy source, which includes light of various wavelengths; when it comes to the application in chemistry, sunlight is primarily used in the field of photocatalysis. Moreover, some studies have indicated that some reactions can be triggered by the ultraviolet ray of sunlight. For example, silicone elastomer could be prepared via thiol-ene click reaction induced by sunlight. ${ }^{33}$ It is an advantage that some reactions can be induced by sunlight because they are "greener" and do not need excessive power as compared to the conventional reactions triggered by a UV light. In this study, the sunlight was applied, for the first time, to trigger the dimerization reaction of the anthryl group. PAPMS- 1 was chosen as an example to design the experiment. The prepolymer PAPMS-1 was prepared and the same amount of the PAPMS-1 solution was diluted to $10 \mathrm{~mL}$ in two colorimetric tubes. One tube was placed under the sunlight and the other was placed indoors. After three days, the samples were tested by UV-vis spectroscopy as illustrated in Fig. 4c. The absorption intensity of the anthracene groups decreased after being irradiated by sunlight, which proved that the dimerization reaction had occurred. Based on these data, it can be concluded that our study can produce the potential self-repair materials by exposure to sunlight.

\section{Luminescent properties of the films}

Anthracene is commonly used in fluorophores that are present in the fluorescent materials and its solution can emit a strong blue light under the UV light irradiation. Simultaneously, anthracene is the exact fetch of most of the fluorescent materials that exhibit aggregation fluorescence quenching (ACQ) effect. They can emit a strong blue light in the solution, but their fluorescence intensity would weaken or even quench in the high-concentration solution or solid. We were surprised to find that PAPMS-1 emitted green fluorescence under a UV light. Thus, we investigated the fluorescence properties of PAPMS-1. It could be observed from Fig. 5a that the fluorescence behavior of PAPMS-1 was similar to that of anthracene. PAPMS-1 can emit a strong blue light in the solution, while the fluorescence intensity weakened or even quenched in the high-concentration solution or the polymer film. Though PAPMS-1 appears like a solid solution, the anthryl group was aggregated due to $\pi-\pi$ stacking. In such case, the fluorescence intensity of the film was much lower than that of the solution. The maximum emission wavelength of the PAPMS-1 film was red shifted to $520 \mathrm{~nm}$ compared with that in the solution $(450 \mathrm{~nm})$. Simultaneously, the cured film was green under a UV light as observed by naked eyes. In PAPMS-1, the anthryl groups were in a dispersed state in the solution. However, they were aggregated owing to $\pi-\pi$ stacking in the films, which led to the red shift of the maximum emission wavelength and exhibited different colors of the fluorescence between the polymer solution and the films. Using the CIE 1931 RGB colorimetric system, the bright emissions of the cured film and the polymer solution can be easily observed by the naked eyes upon excitation with a wavelength of $398 \mathrm{~nm}$ as shown in Fig. 5b.

Interestingly, the fluorescence intensity of the film changed after the irradiation. As shown in Fig. 6, the fluorescence intensity of the PAPMS-1 film increased after the UV-irradiation, which was different from the ACQ effect. The fluorescence emission reduced due to $\pi-\pi$ stacking in the PAPMS- 1 film. The UV-triggered dimerization occurred between the anthracene groups after the irradiation. The $\pi-\pi$ stacking was broken and the aggregated state of the anthracene groups was changed. Thus, the ACQ effect was weakened and the emission increased.

\section{Coating on a commercial available UV-LED light}

The potential application on the commercially available LED cell was determined because of the unique luminescent performance of the PAPMS-1 film. When the PAPMS-1 film was coated
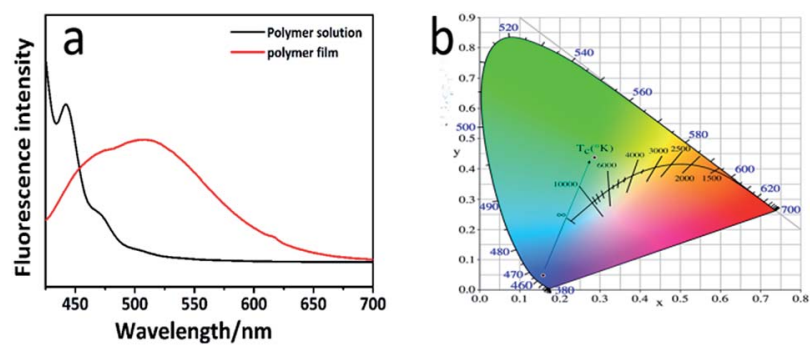

Fig. 5 (a) Fluorescence spectra of the PAPMS-1 film and its solution under UV-irradiation at a $365 \mathrm{~nm}$ wavelength (excitation at $398 \mathrm{~nm}$ ); (b) emission colors of the films in the CIE 1931 chromaticity diagram; CIE color coordinates: polymer solution $(0.1588,0.0478)$ and cured film $(0.3133,0.421)$. 


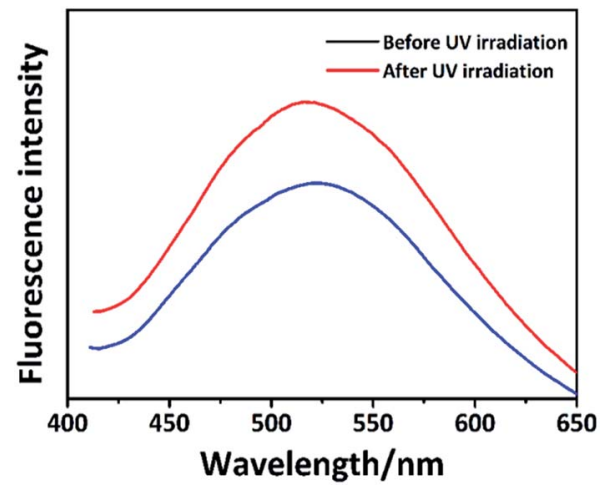

Fig. 6 Fluorescence spectra of the untreated and treated PAPMS-1 film upon UV-irradiation.

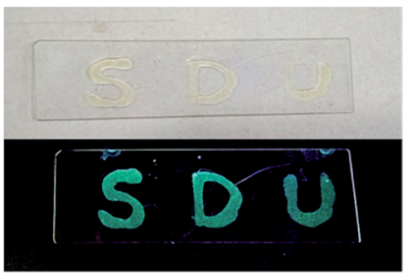

a

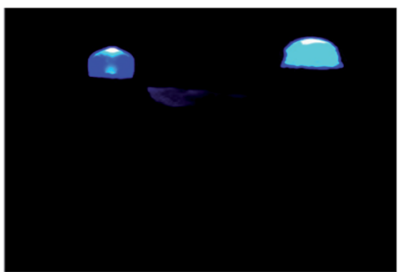

b
Fig. 7 (a) Images of the flat substrate coated with the luminescent film under UV irradiation (365 nm); (b) images of a commercially available UV-LED cell (left) and a UV-LED coated with the luminescent film (right).

on a flat substrate exposed to a UV lamp, a green luminescence was observed even with the naked eyes as illustrated in Fig. 7a. PAPMS-1 has an excellent film-forming ability and can be easily coated on any round-shaped object. Thus, the reactant mixture containing 9-anthracenylmethyl acrylate grafted polysiloxane was also coated onto a commercially available UV-LED cell instead of casting on the flat substrate. Thus, the colorful UVlight emitting diode (LED) cell can be obtained through a facile and efficient method, in which the commercially available LED cell was coated with the transparent luminescent films from the solution medium, followed by in situ curing. As shown in Fig. 7b, a bright green light was observed. We can speculate that if the fluorescent molecular species were changed or the excitation wavelength was adjusted, the change in the emission colors of the LED light could be observed. Therefore, different sorts of colorful LED lights can be obtained via the similar approaches. In addition, the PAPMS-1 film exhibited an excellent thermal stability, which was proven by thermogravimetric analysis (TGA) under $\mathrm{N}_{2}$ (Fig. $\mathrm{S} 8 \dagger$ ). The microphase separation of the PAPMS-1 film was not observed by the scanning electronic microscopy (SEM) as shown in the Fig. S9. $\dagger$

\section{Conclusion}

The synthesis of novel anthracene-containing polysiloxane via aza-Micheal or neutralization reaction and irradiation under a UV light is described in this paper. It is evident that the photocross-linking process is almost reversible, which is proven by the data from the absorption intensity of the UV spectra obtained when the target products were heated. In particular, sunlight can be used instead of UV light to trigger the reaction and it was applied for the first time here in the anthracene dimerization. Moreover, a sunlight-induced reaction is beneficial for the environment. The green luminescent films based on polysiloxane and the anthracene group were developed and possessed the excellent film properties. These transparent films could be coated on a UV-LED cell, which makes them ideal candidates, particularly for fabricating devices used in displaying and lighting in an environment-friendly way.

\section{Conflicts of interest}

There are no conflicts to declare.

\section{Acknowledgements}

This work was financially supported by the National Natural Science Foundation of China (No. 21774070), the National Science Foundation of Shandong Province (ZR2015BQ008), and Shandong Special Fund for Independent Innovation and Achievements transformation (No. 2014ZZCX01101).

\section{References}

1 Y. Zuo, Z. Gou and C. Zhang, Macromol. Rapid Commun., 2016, 37(15), 1052-1059.

2 C. Erbil, E. Kazancıoğlu and N. Uyanık, Eur. Polym. J., 2004, 40(6), 1145-1154.

3 R. P. Eckberg, Ultraviolet light curable acrylic functional silicone compositions, US Pat., US4348454, 1982.

4 S. Matsubara, M. Yoshioka and K. J. Utimoto, Chem. Lett., 1994, 5(5), 827-830.

5 G. Zhiming, Y. Zuo and S. Feng, RSC Adv., 2016, 6(77), 7314073147.

6 H. Lu and S. Feng, J. Polym. Sci., Part A: Polym. Chem., 2017, 55(5), 903-911.

7 Y. Zuo, Z. Gou, J. Cao, et al., RSC Adv., 2016, 6(51), 4519345201.

8 Y. Yue, Y. Liang, H. Wang, L. Feng, S. Feng and H. Lu, Photochem. Photobiol., 2013, 89, 5-13.

9 A. S. Fawcett, T. C. Hughes, L. Zepeda-Velazquez and M. A. Brook, Macromolecules, 2015, 48(18), 6499-6507.

10 A. S. Fawcett and M. A. Brook, Macromolecules, 2014, 47(5), 1656-1663.

11 S. Li and S. Feng, RSC Adv., 2016, 6, 99414-99421.

12 T. Bartz, M. Klapper, K. Müllen and R. C. Schulz, Polym. Int., 1993, 31, 153-162.

13 M. Coursan, J. P. Desvergne and A. Deffieux, Macromol. Chem. Phys., 1996, 197, 1599-1608.

14 J. T. Goldbach, T. P. Russell and J. Penelle, Macromolecules, 2002, 35, 4271-4276.

15 S. Paul, S. Stein, W. Knoll and K. Müllen, Acta Polym., 1996, 47, 92-98. 
16 L. A. Connal, R. Vestberg, C. J. Hawker and G. G. Qiao, Adv. Funct. Mater., 2008, 18, 3315-3322.

17 R. J. Wojtecki, M. A. Meador and S. J. Rowan, Nat. Mater., 2011, 10, 14-27.

18 H. Wang, F. Liu, R. C. Helgeson and K. N. Houk, Angew. Chem., Int. Ed., 2013, 52, 655-659.

19 J. F. Xu, Y. Z. Chen, L. Z. Wu, C. H. Tung and Q. Z. Yang, Org. Lett., 2013, 15, 6148-6151.

20 L. Lopez-Vilanova, I. Martínez, T. Corrales and F. Catalina, Eur. Polym. J., 2014, 56, 69-76.

21 X. A. Trinh, J. Fukuda, Y. Adachi, H. Nakanishi and T. Norisuye, Macromolecules, 2007, 40, 5566-5574.

22 D. Habault, H. Zhang and Y. Zhao, Chem. Soc. Rev., 2013, 42, 7244-7725.

23 H. Xie, C. Y. Cheng, X. Y. Deng, et al., ACS Appl. Mater. Interfaces, 2016, 8, 9431-9439.

24 S. Radl, M. Kreimer, T. Griesser, A. Oesterreicher, A. Moser, W. Kern and S. Schlogl, Polymer, 2015, 80, 76-87.
25 S. Bringmann, R. Brodbeck, R. Hartmann and C. Schafer, Org. Biomol. Chem., 2011, 9(21), 7491-7499.

26 H. Xie, C. Y. Cheng, X. Y. Deng, et al., Macromolecules, 2017, 50, 5155-5164.

27 H. Xie, C. Y. Cheng, L. Du, et al., Macromolecules, 2016, 49, 3845-3855.

28 J. Van Damme, L. laminck, G. Van Assche, et al., Tetrahedron, 2016, 72(29), 4303-4311.

29 T. Yamamoto, S. Yagyu and Y. J. Tezuka, J. Am. Chem. Soc., 2016, 138, 3904-3911.

30 H. D. Becker, Unimolecular photochemistry of anthracenes, Chem. Rev., 1993, 93, 145-172.

31 S. Tazuke and N. Hayashi, Functionality and structure of polymers, J. Polym. Sci., Polym. Chem. Ed., 1978, 16, 27292739.

32 J. Van Damme, O. van den Berg, J. Brancart, et al., Macromolecules, 2017, 50(5), 1930-1938.

33 Y. Zuo, J. Cao and S. Feng, Adv. Funct. Mater., 2015, 25, 27542762. 